\title{
ANTIEMETICS FOR PREVENTION OF NAUSEA AND VOMITING CAUSED BY HIGHLY EMETOGENIC CHEMOTHERAPY: A COCHRANE SYSTEMATIC REVIEW AND NETWORK META-ANALYSIS

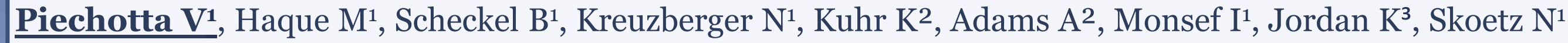 1Department I of Internal Medicine, University of Cologne, Faculty of Medicine and University Hospital Cologne, Cologne, Germany 2Institute of Medical Statistics and Computational Biology, University of Cologne, Faculty of Medicine and University Hospital Cologne, Cologne, Germany

\section{INTRODUCTION}

Combining NK1- and 5-HT3-inhibitors plus dexamethasone is effective in preventing CINV in patients receiving highly emetogenic chemotherapy (HEC)

\section{AIM OF THE STUDY}

to compare different agent-combinations to rank treatments according to efficacy and safety

\section{METHODOLOGY}

- Protocol of this review was previously published in Cochrane Library ${ }^{1}$

- Literature search: Systematic literature search in MEDLINE, CENTRAL, EMBASE, study registries, and conference proceedings (search date 04/2019)

- Inclusion criteria: RCTs including adult cancer patients receiving HEC

- Outcomes of interest:

complete response (CR) in acute (AP), delayed (DP), and overall phase (OP)

$>$ complete control of nausea in AP, DP, OP

$>$ adverse events (AE), on-study mortality (OSM)

quality of life (QoL)

- Quality assessment: Assessed with Cochrane Risk of Bias Tool

- Data analysis:

$>$ Random-effects network meta-analysis (NMA) using frequentist graph-theoretical approach

$>$ Treatment effects: risk ratios (RR) with 95\% confidence intervals $(\mathrm{Cl}) ; \mathrm{RR}>1$ indicates an advantage for the intervention, if not indicated otherwise

> P-scores for treatment ranking: ranging from 0 to 1 ; higher $P$-score indicates a

- Studies were selected, extracted, and evaluated in duplicate.

This project was funded by the Federal Ministry of Education and Research, grant number: 01KG1510 greater chance of being the best treatment

\section{RESULTS}

\section{INCLUDED STUDIES:}

- 50 studies comprising 23,476 patients and 19 treatment regimens (figure 1)

- Overall risk of bias: generally low to unclear across studies

\section{EFFICACY:}

CR:

$>$ Combinations including NK1- and 5 HT3 inhibitors appear to be more effective during AP vs. combinations including only a 5-HT3 inhibitor

$>$ Aprepitant + palonosetron showed highest efficacy vs. granisetron in DP (RR 1.42 [95\% Cl: 1.22-1.65]) and OP (RR: 1.51 [95\% Cl: 1.32-1.74]), cf. figure 2 for all comparisons during DP

No nausea:

$>$ In AP, ezlopitant + granisetron appears to be most effective ( $P$ Score: 0.94)

$>$ In DP palonosetron (RR: 1.37 [95\% Cl: 1.11-1.70]), aprepitant + ondansetron (RR: 1.35 [95\% Cl: 1.06, 1.71]), and rolapitant + granisetron (RR: 1.26 [1.07, 1.47]) showed a higher efficacy vs. granisetron, respectively

$>$ In OP, evidence suggests fosaprepitant + palonosetron to be most effective (P-Score: 0.96)

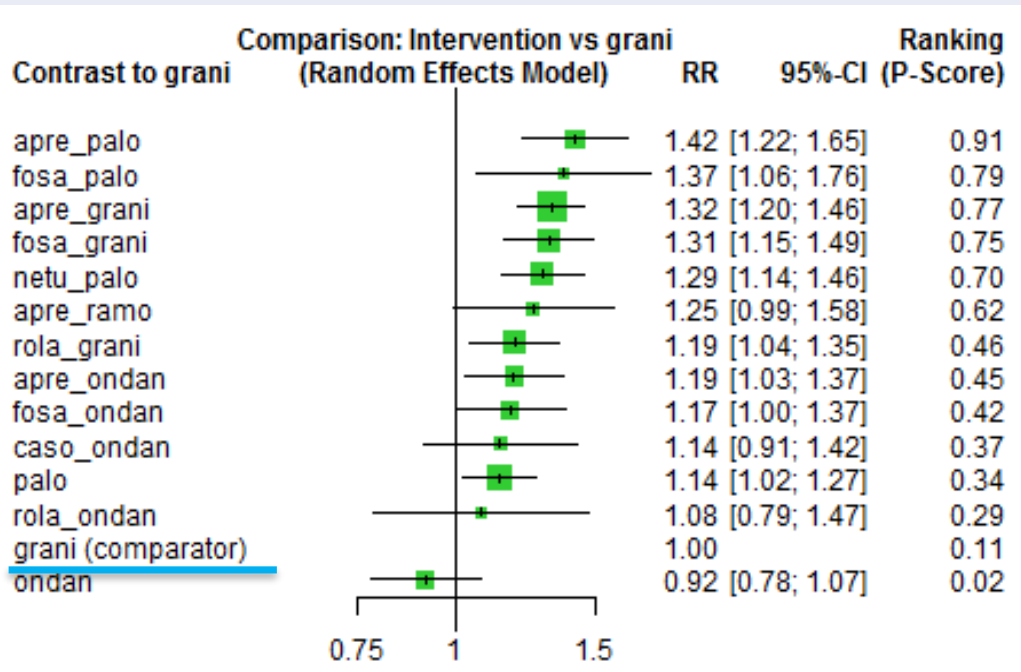

Favours grani Favours intervention

Figure 2: Exemplary NMA forest plot for the outcome CR in DP.

\section{SAFETY:}

QoL:

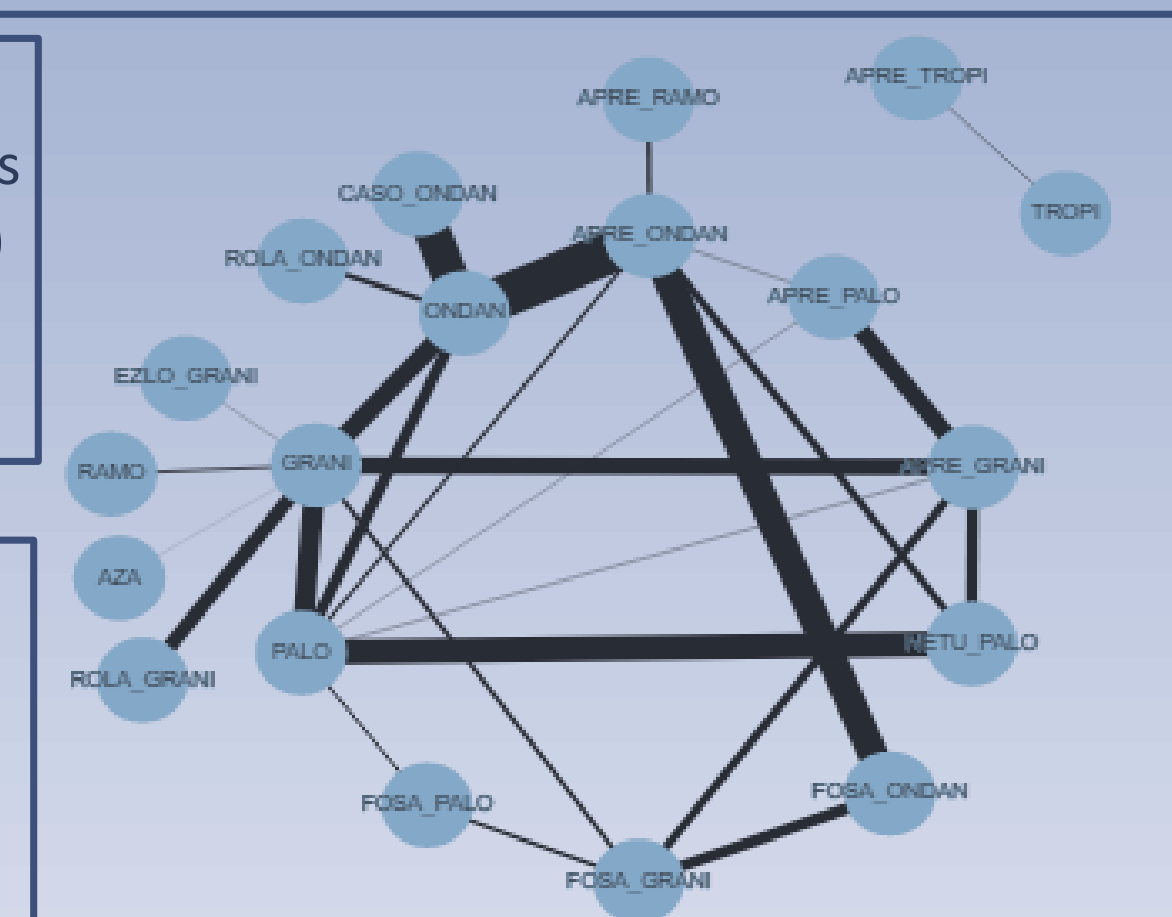

Figure 1: network illustration of the evidence; lines: represents direct comparisons; line widths: number of patients; nodes: treatment

- AEs: aprepitant + granisetron showed a slightly lower risk vs. granisetron (RR: 1.10 [95\% Cl: 1.06-1.14]), cf. figure 3 for all comparisons

- SAEs, Febrile neutropenia, OSM: evidence suggests no differences between combinations

- Neutropenia: aprepitant + ondansetron showed a lower risk vs. ondansetron (RR: 2.56 [95\% Cl: 1.08- 6.07]) and casopitant + ondansetron (RR: 2.84 [95\% Cl: 1.19-6.78]), respectively

- Evidence suggests fewer impairments of QoL for rolapitant + ondansetron vs. ondansetron (RR: 1.46 [95\% Cl: 1.012.10] )

- Evidence suggests no other advantages between combinations

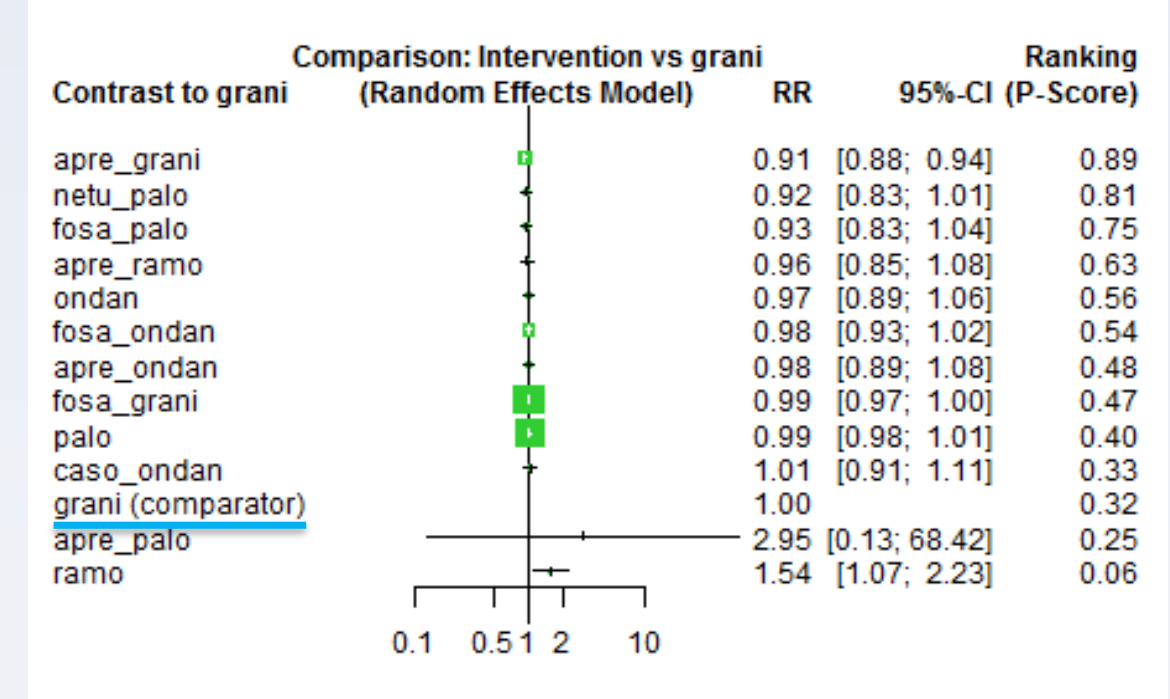

Favours intervention Favours grani

Figure 3: Exemplary NMA forest plot for the outcome AEs.

\section{REFERENCES}

\section{SUMMARY AND CONCLUSIONS}

- This is the first NMA comparing antiemetics for patients receiving HEC

- Aprepitant + palonosetron showed highest CR during DP

- Results may best be confirmed by additional direct comparisons of combinations including NK1- and 5-HT3-inhibitors plus dexamethasone

- Further trials should focus on consistent reporting of safety outcomes and should use a standardized instrument to evaluate QoL to ensure comparability of treatment- 